\title{
Article \\ Modeling and Inversion of Airborne and Semi-Airborne Transient Electromagnetic Data with Inexact Transmitter and Receiver Geometries
}

\author{
Tao Chen ${ }^{1,2}$ and Dikun Yang ${ }^{1,2,3, *(\mathbb{D})}$ \\ 1 Shenzhen Key Laboratory of Deep Offshore Oil and Gas Exploration Technology, Southern University of \\ Science and Technology, Shenzhen 518055, China; chentaosx@hotmail.com \\ 2 Department of Earth and Space Sciences, Southern University of Science and Technology, \\ Shenzhen 518055, China \\ 3 Southern Marine Science and Engineering Guangdong Laboratory (Guangzhou), Guangzhou 511458, China \\ * Correspondence: yangdikun@gmail.com
}

check for

updates

Citation: Chen, T.; Yang, D.

Modeling and Inversion of Airborne and Semi-Airborne Transient Electromagnetic Data with Inexact Transmitter and Receiver Geometries. Remote Sens. 2022, 14, 915. https:// doi.org/10.3390/rs14040915

Academic Editors: Gian Piero Deidda, Mahjoub Himi and Cassiani Giorgio

Received: 17 January 2022

Accepted: 12 February 2022

Published: 14 February 2022

Publisher's Note: MDPI stays neutral with regard to jurisdictional claims in published maps and institutional affiliations.

Copyright: (C) 2022 by the authors. Licensee MDPI, Basel, Switzerland. This article is an open access article distributed under the terms and conditions of the Creative Commons Attribution (CC BY) license (https:// creativecommons.org/licenses/by/ $4.0 /)$.

\begin{abstract}
Airborne and semi-airborne transient electromagnetic (TEM) surveys have high efficiency but may suffer from systematic errors due to the inexact shape, position, and orientation of the transmitter and receiver, which can deviate from the nominal design because of complex terrain, platform instability, or external forces. Without considering actual survey geometry, modeling and inversion can bias the interpretation of results. We develop a universal approach to layered earth capable of modeling arbitrarily complex transmitter and receiver geometry used in airborne and semi-airborne surveys. Our algorithm decomposes an airborne loop or grounded wire source to a set of $x-, y-$, or $z$-oriented electric dipoles. An arbitrarily oriented receiver coil is simulated by projecting three-component data to the actual direction of receiving. In airborne TEM, the transmitter loop and receiver coil are often bound together on a rigid frame and tilt during the flight. Our simulations and synthetic inversion show that such a tilt may reduce responses relative to the data obtained with the nominal geometry; an inversion without considering the tilt can underestimate near-surface conductivity. In semi-airborne TEM, the transmitter wire on the surface can be crooked, and the airborne receiver coil can also tilt. Our modeling shows that the simulated data can change significantly if the actual transmitter and receiver geometry does not exactly follow the nominal survey design; if not appropriately accounted for, such an error may distort the recovered conductivity model. Finally, the benefit of our algorithm is demonstrated by an airborne TEM field data inversion of groundwater problems with the tilt angle of the transmitter-receiver frame accurately modeled. Our work provides a tool for improving the resolution of airborne and semi-airborne TEM in near-surface conductivity characterization.
\end{abstract}

Keywords: transient electromagnetic; airborne; semi-airborne; survey geometry; forward modeling; inversion

\section{Introduction}

Transient electromagnetics (TEM), a time-domain electromagnetic remote sensing technique, has proven to be an effective approach for near-surface geophysical surveys in the past few decades. Its application scenarios include mineral exploration, engineering, hydrology, and environmental investigations [1-5]. With the improvement of the instrument and interpretation, the application scenarios of the TEM approach have been expanded [6,7].

Airborne and semi-airborne TEM are two emerging branches of the TEM method. In airborne TEM (ATEM), the transmitter loop and the receiver coils fly together with the aircraft; the ATEM system transmits electromagnetic signals while measuring the earth's responses in the magnetic field continuously during the flight. In semi-airborne TEM 
(SATEM), the transmitter is a long wire, or a large closed loop fixed to the ground, whereas the receiver coil flies in the air taking magnetic field measurements at a series of locations. It is important to note that electric field measurements on airborne survey platforms are impossible, so the receiver can only sense the magnetic field via a coil or magnetometer. Because of the airborne platform, ATEM and SATEM have high efficiency and mobility compared to ground TEM. They have been shown particularly useful in rapidly assessing extensive or inaccessible areas.

The efficiency of airborne systems comes at the cost of data quality. The bird in ATEM and SATEM is suspended and towed tens of meters above the surface. Nonconstant movement of the aircraft and external forces may cause unpredictable rotation, displacement, and tilt of the bird away from its nominal position and orientation. A large ground loop or long wire is intended to increase the transmitter moment, but long transmitter wires cannot always be deployed along straight lines as planned in practice because of surface obstacles. As a result, the inevitable and sometimes unpredictable deviation of survey configuration in ATEM and SATEM from the nominal parameters can be a significant source of error. This paper refers to such deviation as inexact transmitter and receiver geometry.

An error from inexact geometries is a kind of systematic error or coherent noise, which cannot be easily suppressed by stacking or other statistical methods. In many cases, such errors are considered negligible. However, the demand for high-resolution imaging and quantitative interpretations continues to increase nowadays. Not taking into account the effect of inexact geometry in the inversion may undermine confidence in the interpretation or even produce misleading results. Previous researchers have noticed this problem and proposed solutions. The authors of [8] developed a novel "agree-to-disagree" strategy to identify and correct misrecorded flight height in ATEM. In [9], the authors proposed to record the orientation of the ATEM bird and decompose the tilted loop as a linear combination of three orthogonal magnetic dipoles. The authors of [10] modeled the actual geometry of a large ground loop using a series of electrical dipoles. Generally, it is required to record the actual size, position, and orientation of the transmitter and receiver, and model them by decomposing them into a number of elementary dipoles.

Previous works studied the inexact geometry problem of ATEM and ground large loop TEM and treated them using different dipole source models or data correction methods. Such a problem in SATEM still needs further investigation, as the combination of a grounded long wire source and a drone-borne coil receiver has gained popularity, but the study of such a system is lacking. This paper presents a universal algorithm for both ATEM and SATEM. Our method models an arbitrary 3D source as a weighted sum of small electrical dipoles oriented in the $x-, y-$, and $z$-directions; the tilt of the ATEM and SATEM receiver coil is modeled by projecting three-component $\mathrm{dH} / \mathrm{dt}$ data to the normal direction of the coil; and the forward modeling and sensitivity calculations are carried out using the principle of superposition. Although based on the layered earth model, our improvement advances the technology by providing a unified, convenient, and accurate modeling and inversion framework for ATEM and SATEM data, which are often used by the same group of practitioners but traditionally require separate numerical programs.

In the following, we first set up the physical simulation problem for ATEM and SATEM. Then, the numerical formulas are derived for the semi-analytical solution of the loop source in ATEM and the grounded wire source in SATEM. Using the algorithms, we investigate the effect of inexact geometries in data under different circumstances. In addition, we formulate an inverse problem for ATEM and SATEM based on the Gauss-Newton method and our accurate modeling algorithms. The importance of considering inexact survey geometry in ATEM and SATEM is demonstrated by some synthetic examples. In the end, we show that our methods can make a difference to the inversion and interpretation of field data acquired with inexact survey geometries. 


\section{Inexact Survey Geometry}

Benefitting from the high efficiency and simplicity of 1D modeling, data collected from TEM surveys are frequently forward modeled using a 1D layered earth model. In particular, this kind of model is a sufficient representation of the sedimentary environment and is favored in hydrological and other near-surface investigations. Our development in this work is for the modeling and inversion of the layered earth model in a right-handed Cartesian coordinate system (Figure 1).

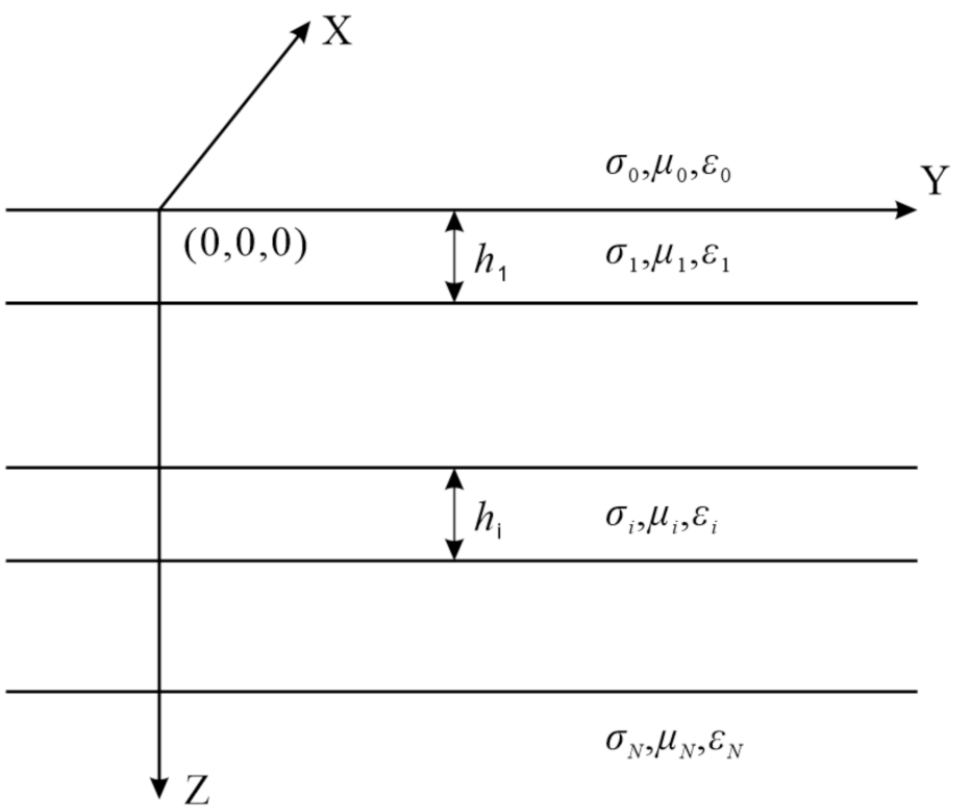

Figure 1. Layered earth model. The physical parameters of each layer are conductivity $(\sigma)$, permeability $(\mu)$, and permittivity $(\varepsilon)$. The thickness of each layer is denoted by $h$. A right-handed Cartesian coordinate system is adopted.

In this paper, we study two airborne variants of TEM methods-ATEM and SATEM surveys. For ATEM, we pay particular attention to systems towed by a helicopter, for example, the SkyTEM and VTEM systems. These systems feature a bird of rigid frame for mounting the transmitter loop and receiver coil (Figure 2). Such a design has been proven advantageous in near-surface and environmental applications that demand highresolution imaging. For SATEM, we consider a current-carrying transmitter wire with its two ends grounded on the surface through a pair of electrodes. Once the ground source is set up, the aircraft tows the receiver coil and flies above the survey area (Figure 2). The transmitters in ATEM and SATEM are sometimes termed the inductive source and galvanic source, respectively; in both cases, the receiver is a horizontal coil that measures the vertical component of the magnetic field or its time derivative. 


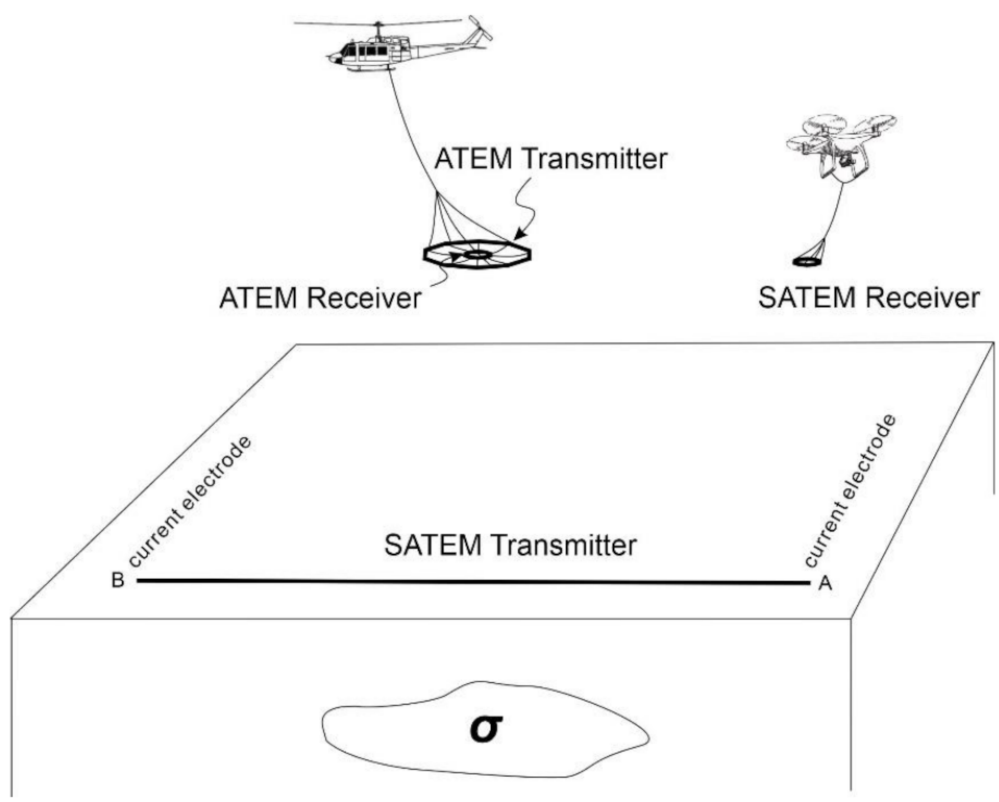

Figure 2. The configuration of ATEM and SATEM systems.

The configurations in Figure 2 are nominal. In practice, ATEM and SATEM always work with inexact survey geometries. Due to various factors such as terrain and wind, the rigid frame of ATEM cannot be exactly leveled as designed (Figure 3a). Modern ATEM systems have been equipped with measuring devices for displacement, tilt angles, and frame clearance. Although this information is often available and delivered to the clients, it is ignored in most cases. SATEM also has the same inexact geometry problem for its receiver coil. In addition, its transmitter wire laid on the surface cannot be guaranteed to be straight when it has to bypass obstacles such as buildings, lakes, roads, etc. (Figure 3b). As a result, the sources of systematic errors in SATEM data can be crooked transmitter wires and tilted receiver coils.

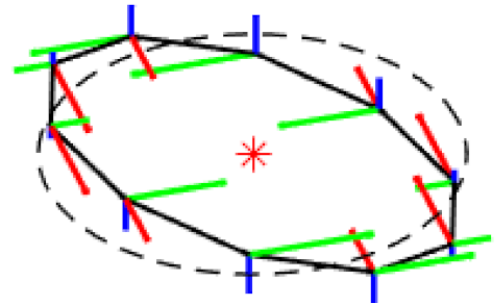

(a)

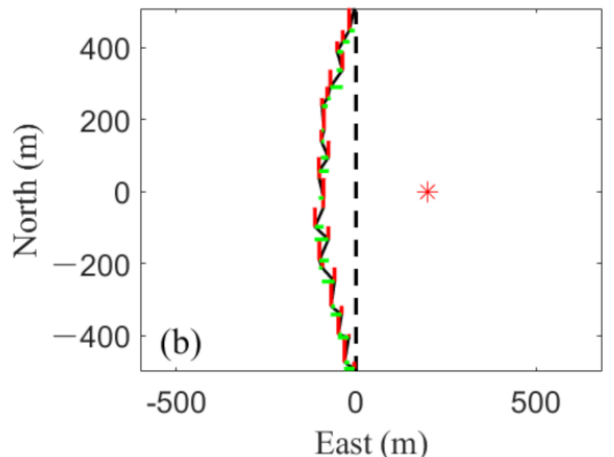

East (m)

Figure 3. Inexact transmitter geometry and its representation with electrical dipoles. (a) ATEM: an airborne transmitter loop tilts from its leveled position (dashed black lines). (b) SATEM: a ground transmitter wire deviates from the straight path (dashed black lines). The solid black indicates the actual transmitter wire path represented by many segments of electric dipoles. The blue, red, and green denote the orthogonal decomposition of each electric dipole to the $x$-, $y$-, and $z$-directions, respectively. The red asterisk represents the receiver.

Fortunately, modern technology such as differential GPS, LiDAR, and the tiltmeter has enabled continuous monitoring of actual survey geometries for airborne systems. The actual way path of the SATEM transmitter wire can also be precisely logged by RTK or other surveying methods. This paper intends to utilize this positioning information to accurately model ATEM and SATEM data and subsequent high-resolution subsurface 
imaging. Here, we propose a universal solution for both ATEM and SATEM. An arbitrary transmitter configuration can be described by a series of waypoints in 3D space and can be simulated using a set of moment-scaled 3D unit electrical dipoles. Each electrical dipole is further decomposed to a linear combination of $x-, y-$, and $z$-oriented dipole sources, which has semi-analytical solutions for layered earth. Figure 3 provides a conceptual diagram using a set of end-to-end electric dipoles to simulate actual transmitter geometry. We note that, in general, the transmitter wire path must be divided finely enough that the distance between the receiver and the dipole is greater than five times the length of the segment [11]. Once the transmitter is decomposed, the original modeling problem becomes many parallel subproblems, each of which is excited by an electrical dipole source in either $x_{-}^{-}, y^{-}$, or $z$-direction. By using the principle of superposition, the modeled data or sensitivity are the sum of these dipole solutions, with the orthogonally projected dipole moments as weights.

The inexact geometry of an airborne receiver is mostly from its rotation or tilt. This paper assumes the receiver coil may have an arbitrary orientation specified by a zenith angle and an azimuthal angle of its normal direction. Our forward-modeled data are always three-component in the $x-, y-$, and $z$-directions; then, the desired data (or sensitivity) are obtained by projecting the modeled vector field data to the actual direction.

\section{Forward Modeling and Analysis}

The core of our modeling method is the computation of the time-domain magnetic field (or its time derivative) responses of layered earth excited by an $x-, y-$, or $z$-oriented electrical dipole source on or above the surface (Figure 3). We first obtained the three-component magnetic field data calculated in the frequency domain. Then, they were frequency-time transformed to the time-domain magnetic field or its time derivative. Finally, convolution with the real transmitter waveform was carried out to obtain the time-domain solution of a specific ATEM or SATEM system.

For a layered earth model, the frequency-domain solution of the magnetic field induced by an $x$-oriented electric dipole (XED) located at $(0,0,-h)$ is [12]

$$
\begin{aligned}
H_{x}^{X E D}= & -\frac{I d s}{2 \pi} \frac{x y}{\rho^{3}} \int_{0}^{\infty}\left(r_{\mathrm{TE}}+r_{\mathrm{TM}}\right) e^{u_{0}(z-h)} J_{1}(\lambda \rho) d \lambda \\
& +\frac{I d s}{4 \pi} \frac{x y}{\rho^{2}} \int_{0}^{\infty}\left(r_{\mathrm{TE}}+r_{\mathrm{TM}}\right) e^{u_{0}(z-h)} \lambda J_{0}(\lambda \rho) d \lambda \\
H_{y}^{X E D}= & -\frac{I d s}{4 \pi}\left(\frac{1}{\rho}-\frac{2 x^{2}}{\rho^{3}}\right) \int_{0}^{\infty}\left(r_{\mathrm{TE}}+r_{\mathrm{TM}}\right) e^{u_{0}(z-h)} J_{1}(\lambda \rho) d \lambda \\
& -\frac{I d s}{4 \pi} \frac{x}{\rho^{2}} \int_{0}^{\infty}\left(r_{\mathrm{TE}}+r_{\mathrm{TM}}\right) e^{u_{0}(z-h)} \lambda J_{0}(\lambda \rho) d \lambda \\
& -\frac{I d s}{4 \pi} \int_{0}^{\infty}\left[e^{-u_{0}(z+h)}-r_{\mathrm{TE}} e^{u_{0}(z-h)}\right] \lambda J_{0}(\lambda \rho) d \lambda \\
H_{z}^{X E D}= & \frac{I d s}{4 \pi} \frac{y}{\rho} \int_{0}^{\infty}\left[e^{-u_{0}(z+h)}+r_{\mathrm{TE}} e^{u_{0}(z-h)}\right] \frac{\lambda^{2}}{u_{0}} J_{1}(\lambda \rho) d \lambda
\end{aligned}
$$

where the subscript of the magnetic field presents the field component, and the superscript of the magnetic field denotes the dipole direction. $(x, y, z)$ are the coordinates of the receiver. $h$ is the height of the dipole above the surface. $\rho$ is the distance between the receiver and dipole. $I d s$ is the transmitter moment. $J_{0}$ and $J_{1}$ are the Bessel functions of the zeroth and first order, respectively. $u_{0}$ is the wavenumber of the air space. $\lambda$ is the integration variable of the Hankel transformation. $r_{\mathrm{TE}}$ is the reflection coefficient for TE mode, and $r_{\mathrm{TM}}$ is the reflection coefficient for TM mode.

The reflection coefficient is defined as

$$
\begin{aligned}
& r_{\mathrm{TE}}=\frac{Y_{0}-\hat{Y}_{1}}{Y_{0}+\hat{Y}_{1}} \\
& \hat{Y}_{n}=Y_{n} \frac{\hat{Y}_{n+1}+Y_{n} \tanh \left(u_{n} h_{n}\right)}{Y_{n}+\hat{Y}_{n+1} \tanh \left(u_{n} h_{n}\right)} \\
& Y_{n}=\frac{u_{n}}{z_{n}} \\
& \hat{Y}_{N}=Y_{N}
\end{aligned}
$$


And

$$
\begin{aligned}
& r_{\mathrm{TM}}=\frac{Z_{0}-\hat{Z}_{1}}{Z_{0}+\hat{Z}_{1}} \\
& \hat{Z}_{n}=Z_{n} \frac{\hat{Z}_{n+1}+Z_{n} \tanh \left(u_{n} h_{n}\right)}{Z_{n}+\hat{Z}_{n+1} \tanh \left(u_{n} h_{n}\right)} \\
& Z_{n}=\frac{u_{n}}{\hat{y}_{n}}=\frac{u_{n}}{i \omega \varepsilon_{n}+\sigma_{n}} \\
& \hat{Z}_{N}=Z_{N}
\end{aligned}
$$

with

$$
\begin{aligned}
& \omega=2 \pi f \\
& u_{n}=\sqrt{\lambda^{2}-k_{n}^{2}} \\
& \hat{z}_{n}=i \omega \mu_{n} \\
& \hat{y}_{n}=i \omega \varepsilon_{n}+\sigma_{n} \\
& k_{n}^{2}=\omega^{2} \mu_{n} \varepsilon_{n}-i \omega \mu_{n} \sigma_{n}
\end{aligned}
$$

where $n$ is the index of the layer. $u_{n}$ is the wavenumber. $h_{n}$ is the thickness. $i$ is the imaginary unit. $\omega$ is the angular frequency and $f$ is the frequency. $k_{n}$ is the vertical wavenumber. $\mu_{0}$ and $\mu_{n}$ are the magnetic permeability of free space and the $n$th layer, respectively.

The magnetic field induced by a $y$-oriented electric dipole (YED) located at $(0,0,-h)$ is [12]

$$
\begin{aligned}
H_{x}^{Y E D}= & -\frac{I d s}{4 \pi}\left(\frac{1}{\rho}-\frac{2 y^{2}}{\rho^{3}}\right) \int_{0}^{\infty}\left(r_{\mathrm{TE}}+r_{\mathrm{TM}}\right) e^{u_{0}(z-h)} J_{1}(\lambda \rho) d \lambda \\
& -\frac{I d s}{4 \pi} \frac{y^{2}}{\rho^{2}} \int_{0}^{\infty}\left(r_{\mathrm{TE}}+r_{\mathrm{TM}}\right) e^{u_{0}(z-h)} \lambda J_{0}(\lambda \rho) d \lambda \\
& -\frac{I d s}{4 \pi} \int_{0}^{\infty}\left[e^{-u_{0}(z+h)}-r_{\mathrm{TE}} e^{u_{0}(z-h)}\right] \lambda J_{0}(\lambda \rho) d \lambda \\
H_{y}^{Y E D}= & -\frac{I d s}{2 \pi} \frac{x y}{\rho^{3}} \int_{0}^{\infty}\left(r_{\mathrm{TE}}+r_{\mathrm{TM}}\right) e^{u_{0}(z-h)} J_{1}(\lambda \rho) d \lambda \\
& +\frac{I d s}{2 \pi} \frac{x y}{\rho^{2}} \int_{0}^{\infty}\left(r_{\mathrm{TE}}+r_{\mathrm{TM}}\right) e^{u_{0}(z-h)} \lambda J_{0}(\lambda \rho) d \lambda \\
H_{z}^{Y E D}= & \frac{I d s}{4 \pi} \frac{y}{\rho} \int_{0}^{\infty}\left[e^{-u_{0}(z+h)}+r_{\mathrm{TE}} e^{u_{0}(z-h)}\right] \frac{\lambda^{2}}{u_{0}} J_{1}(\lambda \rho) d \lambda
\end{aligned}
$$

The magnetic field induced by a z-oriented electric dipole (ZED) located at $(0,0,-h)$ is unavailable from the publicly available literature, so we directly derived them using the Schelkunoff potentials method [11] as follows:

$$
\begin{aligned}
& H_{x}^{Z E D}=-\frac{I d s}{4 \pi} \frac{y}{\rho} \int_{0}^{\infty}\left[e^{-u_{0}(z+h)}+r_{\mathrm{TM}} e^{u_{0}(z-h)}\right] \frac{\lambda^{2}}{u_{0}} J_{1}(\lambda \rho) d \lambda \\
& H_{y}^{Z E D}=\frac{I d s}{4 \pi} \frac{x}{\rho} \int_{0}^{\infty}\left[e^{-u_{0}(z+h)}+r_{\mathrm{TM}} e^{u_{0}(z-h)}\right] \frac{\lambda^{2}}{u_{0}} J_{1}(\lambda \rho) d \lambda \\
& H_{z}^{Z E D}=0
\end{aligned}
$$

Once the frequency-domain responses were obtained, a cosine transform was employed to calculate the time-domain magnetic field and its time derivative induced by a step-off waveform [13].

$$
\begin{aligned}
h(t) & =-\frac{2}{\pi} \int_{0}^{\infty} \frac{\operatorname{Im}[H(\omega)]}{\omega} \cos (\omega t) d \omega \\
\frac{\partial h(t)}{\partial t} & =-\frac{2}{\pi} \int_{0}^{\infty} \operatorname{Re}[H(\omega)] \cos (\omega t) d \omega
\end{aligned}
$$

For efficient computing, we utilized a digital linear filter to calculate the Hankel transform and cosine transform involved in the equations $(1,5,6,7)$. Details about the digital linear filter can be found in a number of publications [14]. When the transmitter waveform is not step off, the program utilizes the convolution of the response induced by a step-off waveform and the derivative of the arbitrary waveform to calculate the response [15].

Our first modeling example concerned a typical central-loop ATEM configuration with a transmitter moment of about $531 \mathrm{~A} \cdot \mathrm{m}^{2}$ and a step-off waveform (Figure $\left.3 \mathrm{a}\right)$. The circular transmitter was approximated by 40 electric dipoles. The zenith angle of the frame 
varied from $5^{\circ}$ to $20^{\circ}$, and the azimuth angle was set to $90^{\circ}$. The flight height was set to $10 \mathrm{~m}$. We compared our accurate modeling with the common practice of simulation using the analytical formula of the central loop configuration for the vertical magnetic field, assuming that the transmitter was a standard circle and was perfectly leveled (the zenith angle is $0^{\circ}$ ). Figure 4 presents the responses over a $0.01 \mathrm{~S} / \mathrm{m}$ half-space. If actual survey geometry is not modeled, the magnitude of data can be reduced by $0.03 \sim 11 \%$. Figure $4 \mathrm{~b}$ also demonstrates that a larger zenith angle, corresponding to a more seriously tilted frame, results in a more pronounced mismatch between the magnitudes of the actual case and the nominal situation. We also found that the discrepancy is most significant in the range of commonly measured time channels, and the late-time responses are more affected.
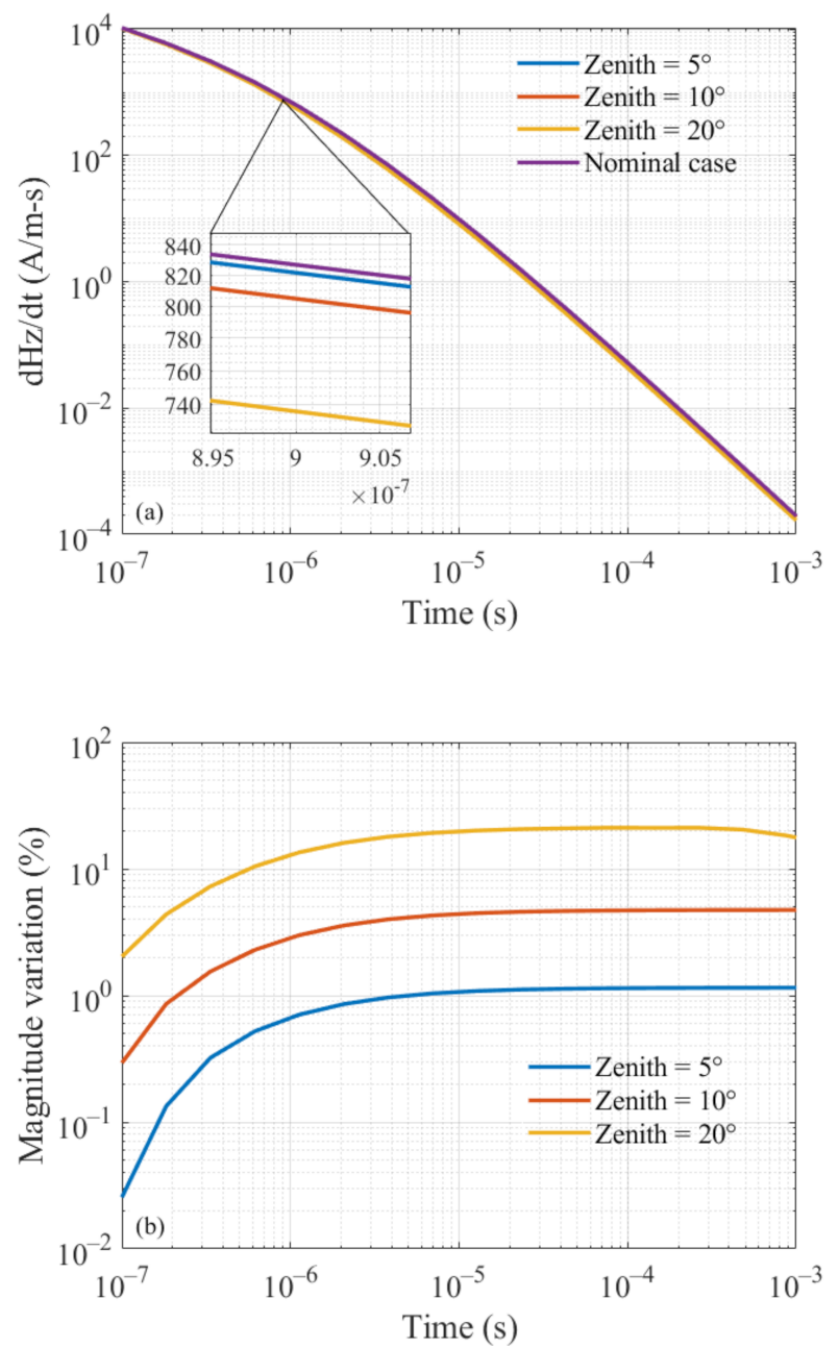

Figure 4. $\mathrm{dHz} / \mathrm{dt}$ response to ATEM survey (central loop configuration) over a half-space with a conductivity of $0.01 \mathrm{~S} / \mathrm{m}$. (a) $\mathrm{dHz} / \mathrm{dt}$ response to the nominal situation and the actual cases where the inexact geometry is considered. (b) mismatch in the magnitude between the nominal situation and the actual cases.

In our second modeling example, we simulated a SATEM survey on a three-layered earth model with conductivity $[0.01,0.1,0.001] \mathrm{S} / \mathrm{m}$ and thickness $[100,100, \infty] \mathrm{m}$. The planned transmitter was a $1000 \mathrm{~m}$ grounded long straight wire, and the waveform was step off. The receiver was located near the center of the source wire, with a $200 \mathrm{~m}$ offset and a height of $10 \mathrm{~m}$ above the surface. In practice, the crooked transmitter was simulated by adding random disturbances to the waypoints of the wire; the maximum deviation was not more than $100 \mathrm{~m}$ (see Figure $3 \mathrm{~b}$ for a plan view). The receiver's zenith and azimuth 
angles were $10^{\circ}$ and $45^{\circ}$, respectively. Errors due to inexact geometries were more complex and more significant for SATEM surveys. If only the transmitter wire was crooked, but the receiver coil was leveled, a large discrepancy could be seen at early times (Figure 5a,b); the errors from a tilted receiver when the transmitter wire was straight were much less significant, but could still reach more than $10 \%$ (Figure $5 c$,d); the compound effect of inexact transmitter and receiver geometries was mostly dominated by the transmitter, with a maximum error of $300 \%$ at early times (Figure 5e,f). In field surveys, it is preferred to place the receiver as close to the source as possible to maximize the received secondary field excited by the transmitter. Our simulation showed that the effect of inexact survey geometries in SATEM can be very strong, especially when the transmitter wire cannot be laid out according to the original plan, but the nominal position is used in the modeling. Proper care is required in the modeling and inversion to prevent the bias from propagating to the inversion models and the geological interpretation.
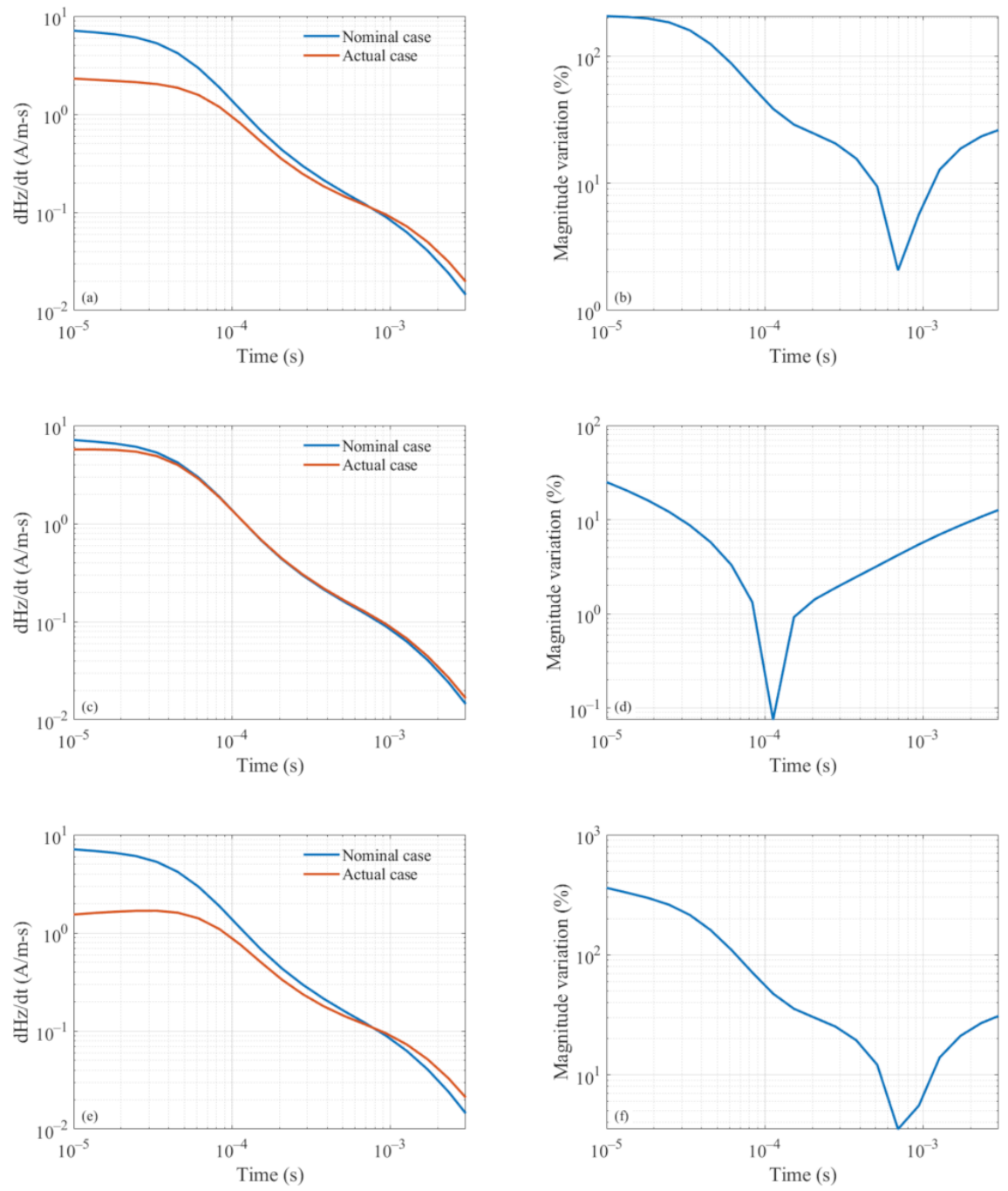

Figure 5. $\mathrm{dHz} / \mathrm{dt}$ responses of the nominal situation and the actual geometry over a three-layered earth model. (a) crooked transmitter only, (c) tilted receiver only, and (e) both crooked transmitter and tilted receiver. $(\mathbf{b}, \mathbf{d}, \mathbf{f})$ show the mismatch in the magnitude for $(\mathbf{a}, \mathbf{c}, \mathbf{e})$, respectively.

\section{Inversion}

In this section, we construct the inverse problem based on the forward modeling approach proposed in the previous section. Using the inversion, we further investigate how 
the effect of inexact survey geometry in ATEM and SATEM can bias the inverted models. In this work, we employ a classical objective function

$$
\phi(\mathbf{m})=\left\|\mathbf{W}_{d}(\mathbf{F}(\mathbf{m})-\mathbf{d})\right\|_{2}^{2}+\mu\left\|\mathbf{W}_{m} \mathbf{m}\right\|_{2}^{2}
$$

where the first term of the right-hand side (RHS) is the data misfit, and the second term is the model norm, which is introduced to stabilize the inverse problem and to add constraints. Specifically, $\mathbf{d}$ represents the measured data, $\mathbf{m}$ is the vector of model parameters, and $\mathbf{F}$ is the nonlinear forward modeling operator presented in Section 3. $\mathbf{W}_{d}$ is the data weighting matrix, which provides the estimation of data uncertainty. If the data error is Gaussian, $\mathbf{W}_{d}$ can be a diagonal matrix whose elements are the reciprocals of the standard deviations of each datum. $\mathbf{W}_{m}$ denotes the model weighting matrix, which can be a thickness weighting operator, first-order difference operator, or focusing operator [16]. A combination of the thickness weighting operator and the first-order difference operator is employed in our implementation. $\mu$ is the regularization parameter, balancing the relative weighting between the data misfit and the model norm during the inversion process. In Equation (8), we use an $L_{2}$ norm to evaluate the data misfit and model norm, but other forms, such as $L_{1}$ norm and $L_{p}$ norm, are alternatives.

We use a Gauss-Newton iterative method to minimize the Equation (8). Because the forward modeling operator $\mathbf{F}$ is nonlinear with respect to $\mathbf{m}$, a Taylor series expansion is utilized to linearize the $\mathbf{F}(\mathbf{m})$ locally as

$$
\mathbf{F}\left(\mathbf{m}_{k+1}\right)=\mathbf{F}\left(\mathbf{m}_{k}\right)+\mathbf{J}_{k}\left(\mathbf{m}_{k+1}-\mathbf{m}_{k}\right)+O\left(\mathbf{m}_{k+1}-\mathbf{m}_{k}\right)
$$

where the $\mathbf{m}_{k+1}$ and $\mathbf{m}_{k}$ are the inverted models at $k+1$ and $k$ th iteration, respectively. $\mathbf{J}_{k}$ represents the Jacobian matrix, the derivative of $\mathbf{d}$ with respect to $\mathbf{m}_{k}$. The iterative equation is found by substituting the Equation (9) into Equation (8), differentiating the Equation (8) with respect to $\mathbf{m}_{k+1}$, and equating it to zeros. Finally, we get the following iterative equation

$$
\begin{array}{r}
{\left[\mathbf{J}_{k}^{T} \mathbf{W}_{d}^{T} \mathbf{W}_{d} \mathbf{J}_{k}+\mu \mathbf{W}_{m}^{T} \mathbf{W}_{m}\right] \mathbf{m}_{k+1}} \\
=\mathbf{J}_{k}^{T} \mathbf{W}_{d}^{T} \mathbf{W}_{d}\left[\mathbf{J}_{k} \mathbf{m}_{k}+\mathbf{d}-\mathbf{F}\left(\mathbf{m}_{k}\right)\right]
\end{array}
$$

The regularization parameter plays an essential role in balancing the data misfit and model norm in the inversion process. Here, we use the following equation to calculate the initial regularization parameter [17]

$$
\mu=\frac{s_{\max }\left(\mathbf{J}_{k}^{T} \mathbf{W}_{d}^{T} \mathbf{W}_{d} \mathbf{J}_{k}\right)}{s_{\max }\left(\mathbf{W}_{m}^{T} \mathbf{W}_{m}\right)}
$$

where $s_{\max }(\mathbf{A})$ denotes the largest singular value of matrix $\mathbf{A}$. In the iterations, a cooling strategy is adopted to update the regularization parameter $\mu$. The iterative process continues until the data misfit has decreased to a specified target (e.g., when the data are assumed to be Gaussian and uncorrelated, an appropriate target is the number of measured data). Alternatively, the inversion can be terminated when the gradient of the objective function falls below a given tolerance [18]. During the inversion, we employed an analytical approach to calculate the Jacobian matrix, which characterizes the sensitivity of the EM fields to changes in layer conductivity [19-21].

The first ATEM synthetic inversion example employed a circular transmitter with an ideal step-off waveform over a three-layered earth model. The receiver was located in the center of the loop, measuring the $z$ component of the $\mathrm{dH} / \mathrm{dt}$ data. The conductivity and thickness of each layer were set as $[0.01,0.002,0.01] \mathrm{S} / \mathrm{m}$ and $[100,200, \infty] \mathrm{m}$, respectively. The transmitter moment was $314.16 \mathrm{~A} \cdot \mathrm{m}^{2}$; the frame's height was $10 \mathrm{~m}$, and there were 20 time channels from $10^{-5} \mathrm{~s}$ to $3 \times 10^{-3} \mathrm{~s}$. In the actual geometry, the zenith and azimuth angles of the receiver coil were $20^{\circ}$ and $90^{\circ}$, respectively. 
Synthetic data were generated by forward modeling with the tilted ATEM frame simulated by our electrical dipole methods. Then the data were inverted by assuming the frame was still perfectly leveled and accurately modeling the actual geometries. Our synthetic test showed that although both inversions achieved a similar level of data fitting and produced a similar structure in the models, noticeable discrepancies in the models were observed in the near-surface and deep layers. Recalling the forward modeling response analysis in Section 3, the discrepancies in both shallow and deep can be explained as the effect of inexact geometry existing in all time channels from early to late. Model bias due to survey geometry does not change qualitative interpretation but may be a problem when high-accuracy results are required in near-surface applications.

The consequences of not modeling actual survey geometry are more prominent for SATEM. Our synthetic test found that inversion with an accurate transmitter wire path and receiver coil orientation can greatly improve the characterization of near-surface structure and enhance the clarity of deep structure compared to an inversion case in which the survey geometry follows the nominal parameters. Although the data fit equally well, the large discrepancy of the models in Figure $6 \mathrm{c}$ reminds us that recording and modeling actual survey geometry can be a critical issue in the processing and interpreting of SATEM data.
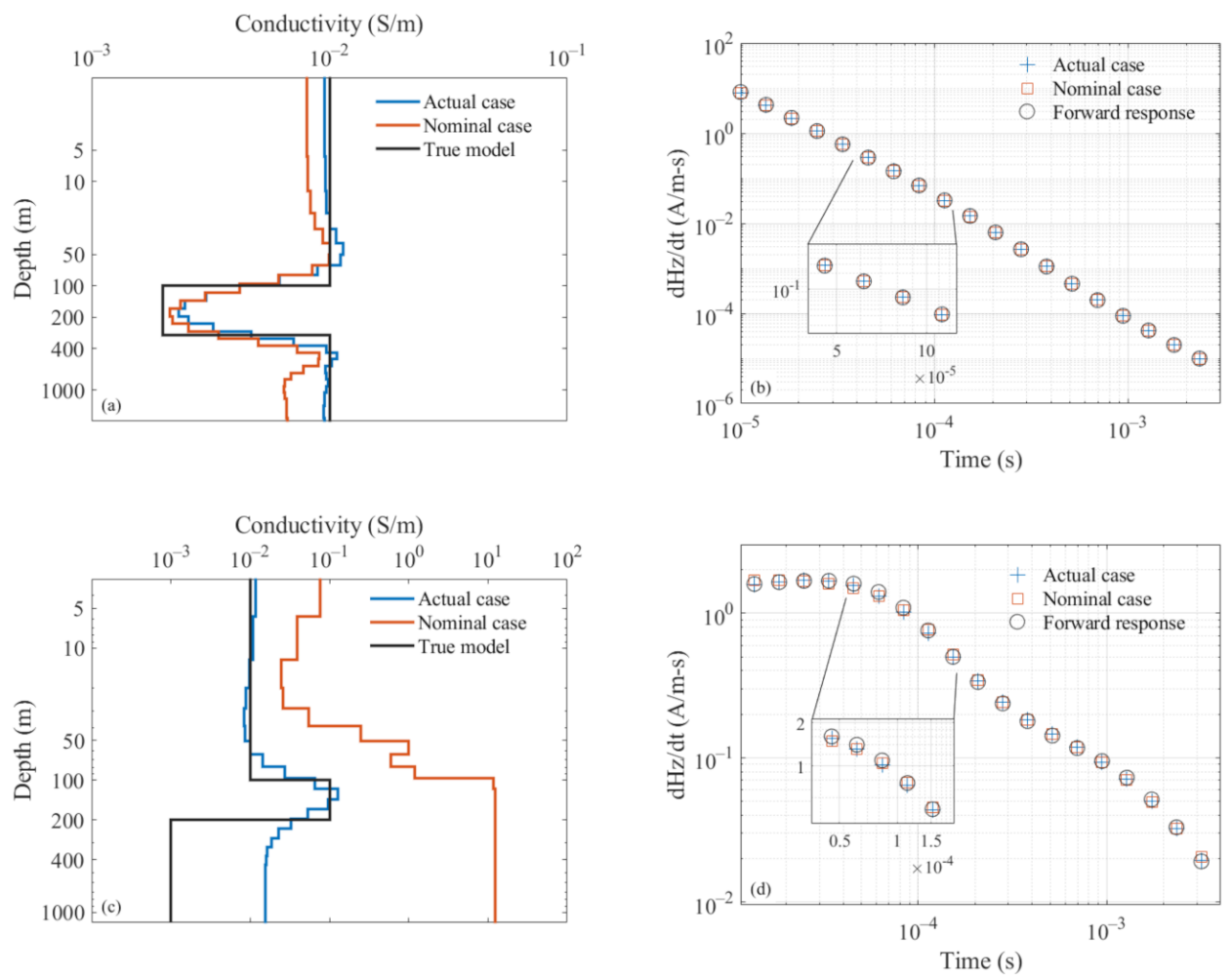

Figure 6. The effect of inexact geometry on the inversion results. (a,b) ATEM: tilted central-loop configuration; (c,d) SATEM: crooked grounded long wire and tilted receiver coil. Detailed survey parameters are presented in Section 3.

Finally, we tested our method in a realistic scenario. This example aimed to demonstrate inversion involving a survey geometry deviating from the nominal. The data were from an ATEM survey of the SkyTEM system collected in 2006 over the Bookpurnong irrigation district in southern Australia. The Murray River and adjacent floodplains in the Bookpurnong area have been extensively salinized. This survey was carried out to map the salinity of the groundwater. We refer readers to [22] for a complete background on the geology and hydrogeology of the study area. The dataset is publicly available in [23]; previous processing and inversions can be found in a number of publications [24-26], in which the tilt of the frame was not considered. 
The SkyTEM operates in high-moment and low-moment modes that are supposed to detect both the deep and shallow. Here we used the low-moment data, which operated at a peak current of $40 \mathrm{~A}$ and a base frequency of $222.5 \mathrm{~Hz}$ [27]. The survey flowed at an altitude of about $60 \mathrm{~m}$ [25]. For the purpose of comparison, we chose one sounding at location $(462,585 \mathrm{~m}, 6,200,782 \mathrm{~m})$, and performed two inversions: one with the actual frame tilt recorded during the survey and the other with the nominal leveled-frame geometry (Figure 7). At that sounding, the zenith and azimuth angles of the frame were about $25^{\circ}$ and $0^{\circ}$, respectively. In general, there was good agreement in the two inversion models, i.e., both of them supported a resistive layer at a depth of about $50 \mathrm{~m}$. However, the near-surface conductivities recovered in the two inversions were evidently different. Not considering the actual tilt of the ATEM frame tended to underestimate the conductivity, and this feature was consistent with that of the synthetic data example. Such a discrepancy in conductivity may lead to non-negligible errors in the interpretation of groundwater salinity.
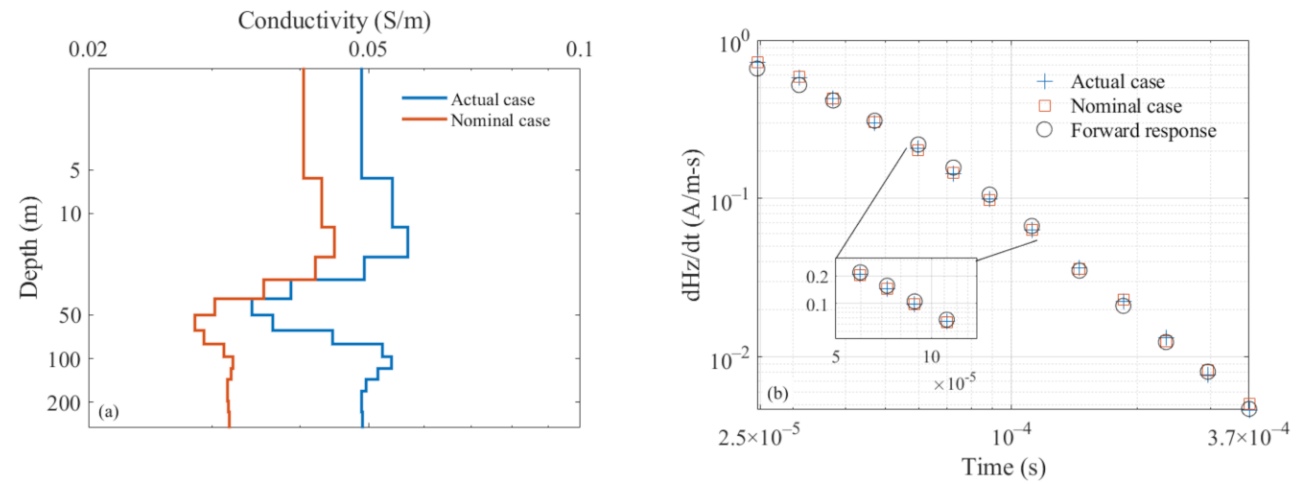

Figure 7. ATEM field data inversions. (a) model recovered with and without actual survey geometry considered. (b) observed and predicted data responses.

SATEM stands out for its cost effectiveness and efficiency and has received more attention in recent years; however, most SATEM systems are still in the experimental stage. To the best of our knowledge, publicly available, reliable SATEM field data are not accessible. Furthermore, in many SATEM surveys, the importance of transmitter and receiver geometry is not realized, and only nominal layouts are reported. Therefore, inversion of SATEM field data taking into account the geometry of the transmitter and receiver is absent in this work. Nonetheless, our work provides theoretical guidance for future SATEM surveys.

\section{Discussion}

Inexact transmitter and receiver geometries are widely present in electromagnetic surveys. However, geometry effects are rarely considered in forward modeling and inversion. The authors of [9] use magnetic dipoles to simulate the geometry of the transmitter in an ATEM survey, yet their application is limited to the closed transmitter coil. The authors of [10] employ $x$ - and $y$-oriented electric dipoles to simulate the ground large loop TEM. However, studies on geometry effects for SATEM survey configurations have not been reported. In this work, we present a universal solution to simulate the geometry of the transmitter. We use electric dipoles (decomposed to $x_{-}, y_{-}$, and $z$-oriented moment-scaled unit electric dipoles) to approximate the geometry of the transmitter, which empower the algorithm to simulate arbitrary geometry configurations in the ATEM and SATEM. We also propose using the superposition principle to calculate the magnetic field in any direction, which often deviates from the nominal case. Its consideration is usually ignored in the SATEM survey. Benefiting from a full consideration of the geometries of the transmitter and receiver, our contribution promotes layered earth simulation by providing a unified and accurate modeling and inversion framework for ATEM, SATEM, and even ground large loop TEM. 


\section{Conclusions}

ATEM and SATEM are often carried out without exactly following the planned transmitter and receiver geometries. In this work, we carefully studied the effect of inexact survey geometry. We developed a unified algorithm that is capable of modeling arbitrary transmitter and receiver configurations in ATEM and SATEM for a layered earth model. The algorithm makes use of superposition by considering a source wire as a combination of unit electrical dipoles in $x-, y$-, or $z$-direction; if the receiver coil is not leveled, the simulated magnetic field vectors need to be projected to the actual receiving direction. Then, a Gauss-Newton inversion method was proposed based on the forward modeling algorithm.

With some synthetic forward and inversion examples, we found that the influence of non-nominal survey geometry in ATEM and SATEM cannot be neglected. In particular, the data errors in SATEM can be as high as a few hundred percent because of a crooked transmitter wire on the ground. Such data errors can then be converted to inaccurately estimated conductivities or even incorrect earth models. In the end, we used a field data example from the Bookpurnong project to show that not recording and modeling actual survey geometry can lead to unreliable interpretation for the evaluation of groundwater salinity. Our work presented in this paper provides the research community with an updated understanding of inexact survey geometry in ATEM and SATEM surveys. The tools we developed here may benefit high-resolution imaging for near-surface characterization.

Author Contributions: Conceptualization, D.Y.; methodology, T.C.; writing-original draft preparation, T.C.; writing-review and editing, T.C. and D.Y.; visualization, T.C.; supervision, D.Y.; project administration, D.Y.; funding acquisition, D.Y. All authors have read and agreed to the published version of the manuscript.

Funding: This work was funded by the Shenzhen Key Laboratory of Deep Offshore Oil and Gas Exploration Technology (Grant No.ZDSYS20190902093007855) and the Key Special Project for Introduced Talents Team of Southern Marine Science and Engineering Guangdong Laboratory (Guangzhou) (GML2019ZD0203).

Institutional Review Board Statement: Not applicable.

Informed Consent Statement: Not applicable.

Data Availability Statement: The data underlying this article will be shared on reasonable request made to the corresponding author.

Acknowledgments: The authors acknowledge Zhitao Xiong and Zhongchang Chen for their discussions on implementing the forward modeling algorithm.

Conflicts of Interest: The authors declare no conflict of interest.

\section{References}

1. Danielsen, J.E.; Auken, E.; Jørgensen, F.; Søndergaard, V.; Sørensen, K.I. The application of the transient electromagnetic method in hydrogeophysical surveys. J. Appl. Geophys. 2003, 53, 181-198. [CrossRef]

2. Fitterman, D.V.; Stewart, M.T. Transient electromagnetic sounding for groundwater. Geophysics 1986, 51, 995-1005. [CrossRef]

3. Li, R.; Yu, N.; Wang, E.; Wang, X.; Mao, L.; Sun, Z. Airborne Transient Electromagnetic Simulation: Detecting Geoelectric Structures for HVdc Monopole Operation. IEEE Geosci. Remote Sens. Mag. 2021, 2-16. [CrossRef]

4. Xue, G.; Qin, K.; Li, X.; Li, G.; Qi, Z.; Zhou, N. Discovery of a large-scale porphyry molybdenum deposit in Tibet through a modified TEM exploration method. J. Environ. Eng. Geophys. 2012, 17, 19-25. [CrossRef]

5. Zhou, N.; Xue, G. Simulation Error Of Rectangular Loop Circular Loop in transient electromagnetic survey. Geophys. Geochem. Explor. 2012, 36, 57-60.

6. Creighton, A.; Parsekian, A.D.; Angelopoulos, M.; Jones, B.M.; Bondurant, A.; Engram, M.; Lenz, J.; Overduin, P.P.; Grosse, G.; Babcock, E.; et al. Transient electromagnetic surveys for the determination of talik depth and geometry beneath thermokarst lakes. J. Geophys. Res. Solid Earth 2018, 123, 9310-9323. [CrossRef]

7. Minami, T.; Toh, H.; Ichihara, H.; Kawashima, I. Three-Dimensional Time Domain Simulation of Tsunami-Generated Electromagnetic Fields: Application to the 2011 Tohoku Earthquake Tsunami. J. Geophys. Res. Solid Earth 2017, 122, 9559-9579. [CrossRef] 
8. Chen, T.; Yang, D. Joint Inversion of Time-Domain and Frequency-Domain Electromagnetic Data Contaminated by Coherent Noises Using an Agree-to-Disagree Strategy. SEG Technical Program Expanded Abstracts 2020; Society of Exploration Geophysicists: Houston, TX, USA, 2020; pp. 565-569.

9. Qi, Y.; Huang, L.; Wang, X.; Fang, G.; Yu, G. Airborne transient electromagnetic modeling and inversion under full attitude change. IEEE Geosci. Remote Sens. Lett. 2017, 14, 1575-1579. [CrossRef]

10. Li, H.; Xue, G.; Zhao, P.; Zhou, N.; Zhong, H. Inversion of arbitrary segmented loop source TEM data over a layered earth. J. Appl. Geophys. 2016, 128, 87-95. [CrossRef]

11. Ward, S.H.; Hohmann, G.W. Electromagnetic theory for geophysical applications. In Electromagnetic Methods in Applied Geophysics; Society of Exploration Geophysicists: Houston, TX, USA, 1988; Volume 1, pp. 130-311.

12. Singh, N.P.; Mogi, T. EMDPLER: A F77 program for modeling the EM response of dipolar sources over the non-magnetic layer earth models. Comput. Geosci. 2010, 36, 430-440. [CrossRef]

13. Newman, G.A.; Hohmann, G.W.; Anderson, W.L. Transient electromagnetic response of a three-dimensional body in a layered earth. Geophysics 1986, 51, 1608-1627. [CrossRef]

14. Werthmüller, D.; Key, K.; Slob, E.C. A tool for designing digital filters for the Hankel and Fourier transforms in potential, diffusive, and wavefield modeling. Geophysics 2019, 84, F47-F56. [CrossRef]

15. Yin, C.; Huang, W.; Ben, F. The full-time electromagnetic modeling for time-domain airborne electromagnetic systems. Chin. J. Geophys. 2013, 56, 3153-3162.

16. Zhdanov, M.S.; Ellis, R.; Mukherjee, S. Three-dimensional regularized focusing inversion of gravity gradient tensor component data. Geophysics 2004, 69, 925-937. [CrossRef]

17. Grayver, A.V.; Streich, R.; Ritter, O. Three-dimensional parallel distributed inversion of CSEM data using a direct forward solver Geophys. J. Int. 2013, 193, 1432-1446. [CrossRef]

18. Pidlisecky, A.; Haber, E.; Knight, R. RESINVM3D: A 3D resistivity inversion package. Geophysics 2007, 72, H1-H10. [CrossRef]

19. Constable, S.C.; Parker, R.L.; Constable, C.G. Occam's inversion: A practical algorithm for generating smooth models from electromagnetic sounding data. Geophysics 1987, 52, 289-300. [CrossRef]

20. Key, K. 1D inversion of multicomponent, multifrequency marine CSEM data: Methodology and synthetic studies for resolving thin resistive layers. Geophysics 2009, 74, F9-F20. [CrossRef]

21. Ogunbo, J.N. Computation of analytical sensitivity matrix for the frequency-domain EM data: MATLAB code. Comput. Geosci. 2018, 117, 41-48. [CrossRef]

22. Munday, T.J.; Doble, R.; Berens, V.; Fitzpatrick, A. The Application of Air, Ground and In River' Electromagnetics in the Definition of Spatial Patterns of Groundwater Induced Salt Accumulation in a Salinising Floodplain, Lower River Murray, South Australia. In Proceedings of the Symposium on the Application of Geophysics to Engineering and Environmental Problems, Seattle, WA, USA, 2-6 April 2006; pp. 886-891. Available online: https://www.earthdoc.org/content/papers/10.3997/2214-4609-pdb.181.93 (accessed on 10 February 2022).

23. Heagy, L.J.; Cockett, R.; Kang, S.; Rosenkjaer, G.K.; Oldenburg, D.W. A framework for simulation and inversion in electromagnetics. Comput. Geosci. 2017, 107, 1-19. [CrossRef]

24. Viezzoli, A.; Auken, E.; Munday, T. Spatially constrained inversion for quasi 3D modelling of airborne electromagnetic data-an application for environmental assessment in the Lower Murray Region of South Australia. Explor. Geophys. 2009, 40, 173-183. [CrossRef]

25. Viezzoli, A.; Munday, T.; Auken, E.; Christiansen, A.V.; Wilson, G.A. Accurate quasi 3D versus practical full 3D inversion of AEM data-the Bookpurnong case study. Preview 2010, 149, 23-31. [CrossRef]

26. Wilson, G.A.; Cox, L.H.; Zhdanov, M.S. Practical 3D inversion of entire airborne electromagnetic surveys. Preview 2010, 146, 29-33.

27. Sørense, K.I.; Auken, E. SkyTEM? A new high-resolution helicopter transient electromagnetic system. Explor. Geophys. 2004, 35, 194-202. [CrossRef] 